\title{
Charged current neutrino induced coherent pion production
}

\author{
L. Alvarez-Ruso ${ }^{a}$, L. S. Geng ${ }^{a}$, S. Hirenzaki ${ }^{b}$ and M. J. Vicente Vacas $^{a}$ \\ ${ }^{a}$ Departamento de Física Teórica and IFIC, Universidad de Valencia - CSIC; \\ Institutos de Investigación de Paterna, Aptdo. 22085, 46071 Valencia, Spain. \\ ${ }^{b}$ Department of Physics, Nara Women's University, Nara, 630-8506, Japan.
}

\begin{abstract}
We analyze the neutrino induced charged current coherent pion production at the energies of interest for recent experiments like K2K and MiniBooNE. Medium effects in the production mechanism and the distortion of the pion wave function, obtained solving the Klein Gordon equation with a microscopic optical potential, are included in the calculation. We find a strong reduction of the cross section due to these effects and also substantial modifications of the energy distributions of the final lepton and pion.
\end{abstract}

\section{INTRODUCTION}

Recent experimental results from the K2K collaboration show a significant deficit of muons in the forward scattering events with respect to the simulations, which could limit the accuracy of the predicted neutrino energy spectrum at the far detector [1]. This deficit could be due, among other possibilities, to the overestimation of charged current (CC) coherent pion production. A later search [2] found no evidence of $\mathrm{CC}$ coherent pion production in ${ }^{12} \mathrm{C}$ and obtained an upper limit for the fraction of this process to the total $\mathrm{CC}$ interaction well below some estimations based on the Rein and Sehgal model of Ref. 3]. Preliminary MiniBooNE results for neutral current $\pi^{0}$ production show a similar deficit [4] in the forward direction when compared with different MonteCarlo (MC) models: NUANCE [5], NEUGEN [6] and NEUT [7]. The discrepancies between these MC simulations indicate the considerable uncertainties in the theoretical description of coherent pion production. In addition, MiniBooNE has collected a large set of data for $\pi^{+}$production in ${ }^{12} \mathrm{C}$ induced by muon neutrinos of energies around $0.7 \mathrm{GeV}$ [8]. A fraction of these pions is created coherently, so that a realistic description of the coherent process is required to analyze and understand these data.

$\mathrm{CC}$ coherent pion production has been observed experimentally at higher energies and for several nuclei 9 , 10, 11, 12, 13, 14]. These experiments were studied theoretically using models based on PCAC [3, 15] which described well the results. Work along the same lines has been carried out at low energies in Ref. [16]. The only nuclear medium effect considered in these calculations is the distortion of the final pion.

Other approaches have tried to incorporate some additional nuclear medium effects that modify the weak pion production. In Ref. 17], the authors used the impulse approximation with undistorted pion waves, but already modified the $\Delta$ resonance properties, and therefore the production mechanisms, using an effective $\Delta$ mass. The importance of these nuclear effects was demonstrated there by comparing the results with those obtained using a free $\Delta$. Kelkar et al. [18] developed a more sophisticated treatment of the $\Delta$ in the nuclear medium, and also included the final pion distortion by solving the Klein Gordon (KG) equation with a pion nucleus optical potential. This model predicted a very low cross section, compatible with the recent results of Ref. [2]. Nonetheless, there were several approximations in this work. On the one side a non relativistic reduction of the hadronic current was done, on the other side all the transverse parts of the amplitude were neglected. Whereas these approximations are quite reasonable to get an estimate of the cross section for this process, as discussed in Ref. 18], a more complete calculation is required now that new data are becoming available. More recently, Singh et al. 19, 20] used similar medium effects on the production mechanisms and improved on the description of the elementary $\nu+N \rightarrow N+\mu^{-}+\pi^{+}$process by using a fully relativistic calculation of this process and including all pieces of the amplitude. However, the pion distortion was implemented in the eikonal approximation, which is not very reliable for the low energy pions that apparently dominate this reaction. Our aim in this paper is to improve the model of Kelkar et al. [18] in a similar manner, using a more complete and relativistic elementary amplitude but still keeping a more realistic treatment of the pion distortion which is calculated solving the KG equation.

In the following, we describe the formalism, including $\Delta$ production and decay, medium effects on the production mechanism and the optical potential responsible for the distortion of the pion. In Section [II we present our results, compare with the available experimental data and make predictions for other nuclei and observables. Finally, our summary and conclusions appear in Section IV. 


\section{THEORETICAL MODEL}

In the CC coherent pion production induced by neutrinos $\left(\nu+A \rightarrow A+\mu^{-}+\pi^{+}\right)$, the nucleus remains in its ground state. The process consists of a weak pion production followed by the strong distortion of the pion in its way out of the nucleus. First, we must consider the elementary process of $\pi^{+}$production $\left(\nu+N \rightarrow \mu^{-}+N+\pi^{+}\right)$. There are large experimental uncertainties in this cross section at the low energies relevant to this work, which can be clearly appreciated by comparing, for instance, Refs. 21] and 22]. These discrepancies are ultimately translated into different values for the $\Delta$ resonance axial form factors that appear in the theoretical models, and are a source of uncertainty for the calculation of the pion production cross sections. In any case, it seems to be clear that for neutrino energies below $2 \mathrm{GeV}$, this process is dominated by a $\Delta(1232)$ excitation, $\nu+p \rightarrow \mu^{-}+\Delta^{++}$or $\nu+n \rightarrow \mu^{-}+\Delta^{+}$ followed by its decay 23, 24, 25, 26, 27, 28, 29], and therefore it is larger for protons than for neutrons as discussed below. The situation is different for $\pi^{0}$ production or at higher energies. In that case, the $\Delta$ mechanism is not enough to provide a good description of data [29]. After the introduction of the elementary model for the reaction we discuss how it is modified in the nuclear medium, due to the density dependent changes of the $\Delta$ resonance peak's position and width. Finally, we study the distortion of the pion wave function using a pion nucleus optical potential.

\section{A. $\Delta$ production and decay}

The matrix element for the elementary process $\nu_{\mu}(k)+n(p) \rightarrow \Delta^{+}\left(p^{\prime}\right)+\mu^{-}\left(k^{\prime}\right)$ is written as

$$
\mathcal{M}_{n, \Delta^{+}}=\frac{G}{\sqrt{2}} \cos \theta_{c} l_{\alpha} J_{\Delta}^{\alpha}
$$

with the leptonic current

$$
l_{\alpha}=\bar{u}_{\ell}\left(k^{\prime}\right) \gamma_{\alpha}\left(1-\gamma_{5}\right) u_{\nu_{\ell}}(k),
$$

and the hadronic current

$$
\begin{gathered}
J_{\Delta}^{\alpha}=\bar{\psi}_{\mu}\left(p^{\prime}\right) \mathcal{A}^{\mu \alpha} u(p), \\
\mathcal{A}^{\mu \alpha}=\left\{\frac{C_{3}^{V}}{M}\left(g^{\mu \alpha} \not q-q^{\mu} \gamma^{\alpha}\right)+\frac{C_{4}^{V}}{M^{2}}\left(g^{\mu \alpha} q \cdot p^{\prime}-q^{\mu} p^{\prime \alpha}\right)+\frac{C_{5}^{V}}{M^{2}}\left(g^{\mu \alpha} q \cdot p-q^{\mu} p^{\alpha}\right)\right\} \gamma_{5} \\
+\left\{\frac{C_{3}^{A}}{M}\left(g^{\mu \alpha} \not q-q^{\mu} \gamma^{\alpha}\right)+\frac{C_{4}^{A}}{M^{2}}\left(g^{\mu \alpha} q \cdot p^{\prime}-q^{\mu} p^{\prime \alpha}\right)+C_{5}^{A} g^{\mu \alpha}+\frac{C_{6}^{A}}{M^{2}} q^{\mu} q^{\alpha}\right\}
\end{gathered}
$$

where $M$ is the nucleon mass, $\psi_{\mu}\left(p^{\prime}\right)$ and $u(p)$ are the Rarita Schwinger and Dirac spinors for the $\Delta$ and the nucleon of momentum $p^{\prime}$ and $p, q=p^{\prime}-p=k-k^{\prime}$ is the momentum transfer, $C_{i}^{V}$ and $C_{i}^{A}(i=3,4,5,6)$ are the vector and axial vector transition form factors. The amplitude for the process on protons, $\nu_{\mu}(k)+p(p) \rightarrow \Delta^{++}\left(p^{\prime}\right)+\mu^{-}\left(k^{\prime}\right)$, is related to the previous one by an isospin factor

$$
\mathcal{M}_{p, \Delta^{++}}=\sqrt{3} \mathcal{M}_{n, \Delta^{+}} .
$$

The vector form factors can be related to the electromagnetic ones. The conservation of the vector part of the current implies that $C_{6}^{V}=0$. The assumption of $M_{1+}$ dominance for the $\Delta$ electroproduction amplitude gives [23]

$$
C_{5}^{V}=0, C_{4}^{V}=-\frac{M}{M_{\Delta}} C_{3}^{V}
$$

and for $C_{3}^{V}$ we take [30]

$$
C_{3}^{V}=\frac{2.05}{\left(1-q^{2} / 0.54 \mathrm{GeV}^{2}\right)^{2}} .
$$

Except for $C_{6}^{A}$ that can be related to $C_{5}^{A}$ using PCAC, there are no other constraints for the axial form factors. We use the following ones, fitted to neutrino scattering data 21, 26, 30, 31, 32, 33, 34, 35, 36, 37]

$$
C_{i=3,4,5}^{A}\left(q^{2}\right)=C_{i}^{A}(0)\left[1-\frac{a_{i} q^{2}}{b_{i}-q^{2}}\right]\left(1-\frac{q^{2}}{M_{A}^{2}}\right)^{-2}
$$


and

$$
C_{6}^{A}\left(q^{2}\right)=C_{5}^{A} \frac{M^{2}}{m_{\pi}^{2}-q^{2}},
$$

with $C_{3}^{A}(0)=0, C_{4}^{A}(0)=-0.3, C_{5}^{A}(0)=1.2, a_{4}=a_{5}=-1.21, b_{4}=b_{5}=2 \mathrm{GeV}^{2}$ and for the axial mass we take $M_{A}=1.28 \mathrm{GeV}$ [32]. This set of form factors produces a good agreement with weak $\Delta$ production data induced by neutrinos on nucleons [26].

A new analysis of world electron scattering data [38] allows to go beyond the $M_{1+}$ approximation and update the vector form factors [39]. This information calls for a new extraction of the axial form factors. Some steps in this direction have been recently taken in Refs. 27, 40], using models for the elementary reaction which include, apart from $\Delta$ excitation, some background terms. For the sake of consistency with our description of pion production on the nucleon explained previously, based on the $\Delta$ dominance, we stick to the set of form factors given above.

We still need to take into account the $\Delta$ decay into a pion and a nucleon. We use the following Lagrangian to describe the $\Delta N \pi$ transition [41]

$$
\mathcal{L}_{\Delta \pi N}=\frac{f^{*}}{m_{\pi}} \bar{\Psi}_{\mu} \vec{T}^{\dagger} \cdot \partial^{\mu} \vec{\Phi} \Psi+\text { h.c. },
$$

where $\vec{T}^{\dagger}$ is the isospin $1 / 2$ to $3 / 2$ transition operator and $\Psi_{\mu}, \vec{\Phi}$ and $\Psi$ are the $\Delta$, pion and nucleon fields respectively. The isospin operator produces a factor 1 for the decay $\Delta^{++} \rightarrow \pi^{+}+p$ and a factor $1 / \sqrt{3}$ for the decay $\Delta^{+} \rightarrow \pi^{+}+n$. This, together with Eq. (5), implies that the amplitude for $\mathrm{CC} \pi^{+}$production on the proton is three times larger than on the neutron. The coupling constant $f^{*}=2.13$ is such that the experimental $\Delta \rightarrow \pi N$ width is reproduced. At the high $\Delta$ invariant masses reached with the K2K or MiniBooNE neutrino energies, the finite size of the hadrons becomes relevant. This can be empirically taken into account with a form factor $F\left(p^{\prime}\right)$ that modifies the $\Delta N \pi$ coupling. Here, we adopt the following ansatz

$$
F\left(p^{\prime}\right)=\frac{\Lambda^{4}}{\Lambda^{4}+\left(p^{\prime 2}-M_{\Delta}^{2}\right)^{2}}
$$

with $\Lambda=1 \mathrm{GeV}$, used in coupled channel studies of pion and photoproduction of baryonic resonances [42]. The new hadronic current which should replace $J_{\Delta}^{\alpha}$ in Eq. (1), already incorporating the decay into a pion and a nucleon, is given (for the case of $\Delta^{+}$) by

$$
J_{N \pi}^{\mu}=-\frac{1}{\sqrt{3}} \frac{f^{*}}{m_{\pi}} p_{\pi}^{\alpha} F\left(p^{\prime}\right) \bar{u}\left(p_{f}\right) D\left(p^{\prime}\right) \Lambda_{\alpha \beta} \mathcal{A}^{\beta \mu} u(p),
$$

where $p_{\pi}$ and $p_{f}$ are the pion and final nucleon momenta so that $p^{\prime}=p_{\pi}+p_{f}$. The $\Delta$ propagator is given by

$$
D\left(p^{\prime}\right)=\frac{1}{\left(W+M_{\Delta}\right)\left(W-M_{\Delta}+i \Gamma_{\Delta} / 2\right)}
$$

where $W=\sqrt{p^{\prime 2}}$. The energy dependent $\Delta$ width is

$$
\Gamma_{\Delta}=\frac{1}{6 \pi}\left(\frac{f^{*}}{m_{\pi}}\right)^{2} F\left(p^{\prime}\right)^{2} \frac{M_{\Delta}}{W} p_{\pi, c m}^{3},
$$

with $p_{\pi, c m}$ the pion momentum in the $\Delta$ rest frame. Finally, the spin $3 / 2$ projection operator is given by

$$
\Lambda_{\alpha \beta}=-\left(\not p^{\prime}+M_{\Delta}\right)\left(g_{\alpha \beta}-\frac{2}{3} \frac{p_{\alpha}^{\prime} p_{\beta}^{\prime}}{M_{\Delta}^{2}}+\frac{1}{3} \frac{p_{\alpha}^{\prime} \gamma_{\beta}-p_{\beta}^{\prime} \gamma_{\alpha}}{M_{\Delta}}-\frac{1}{3} \gamma_{\alpha} \gamma_{\beta}\right)
$$

\section{B. $\Delta$ in the nuclear medium}

The $\Delta$ properties are strongly modified inside the nuclear medium and have been the subject of intensive study, both experimental and theoretical, for many years, see i.e. [43, 44, 45, 46, 47, 48, 49, 50] and references therein. In this work we use the results from Refs. [50, 51] where the $\Delta$ selfenergy is calculated in a many body approach as a function of the local baryon density $\rho(r)$. This model has been extensively tested in pion induced inclusive processes [52, 53], 
photonuclear reactions [54, 55], electron scattering [56] and even for coherent pion production induced by photons, electrons or nuclei [57, [58, [59, 60]. In the nuclear medium the $\Delta$ resonance acquires a selfenergy because of several effects such as Pauli blocking of the final nucleon and absorption processes: $\Delta N \rightarrow N N, \Delta N \rightarrow N N \pi$ or $\Delta N N \rightarrow$ $N N N$. The real part can be parametrized as

$$
\operatorname{Re} \Sigma_{\Delta}(\rho)=\operatorname{Re} \Sigma_{\Delta}^{0}(\rho)+\frac{4}{9}\left(\frac{f^{*}}{m_{\pi}}\right)^{2} g^{\prime} \rho \approx 40 \mathrm{MeV} \frac{\rho}{\rho_{0}}
$$

where $\rho_{0}=0.17 \mathrm{fm}^{-3}$ is the normal nuclear density. Here, in addition to the attractive proper selfenergy $\operatorname{Re} \Sigma_{\Delta}^{0}(\rho)$, the effective repulsive contribution, that comes from the iterated $\Delta$-hole excitation driven by the Landau Migdal interaction with $g^{\prime}=0.63$, has been added [54]. The imaginary part is parametrized by the expression

$$
-\operatorname{Im} \Sigma_{\Delta}(\rho)=C_{Q}\left(\frac{\rho}{\rho_{0}}\right)^{\alpha}+C_{A 2}\left(\frac{\rho}{\rho_{0}}\right)^{\beta}+C_{A 3}\left(\frac{\rho}{\rho_{0}}\right)^{\gamma}
$$

where the terms with the coefficients $C_{Q}, C_{A 2}$ and $C_{A 3}$ correspond to the processes $\Delta N \rightarrow N N \pi, \Delta N \rightarrow N N$ and $\Delta N N \rightarrow N N N$ respectively. The values of $C_{Q}, C_{A 2}, C_{A 3}, \alpha, \beta$ and $\gamma$ can be found in Eq. (4.5) and Table 2 of Ref. [52]. The parameterizations are given as a function of the kinetic energy in the laboratory system of a pion that would excite a $\Delta$ with the corresponding invariant mass, and are valid in the range $85 \mathrm{MeV}<T_{\pi}<315 \mathrm{MeV}$. Below $85 \mathrm{MeV}$ the contributions from $C_{Q}$ and $C_{A 3}$ are rather small and are taken from [51], where the model was extended to low energies. The term with $C_{A 2}$ shows a very mild energy dependence and we still use the parameterization from Ref. 52 ] even at low energies. For $T_{\pi}$ above $315 \mathrm{MeV}$ we have kept these selfenergy terms constant and equal to their values at the bound. The uncertainties in these pieces are not very relevant there because the $\Delta \rightarrow N \pi$ decay becomes very large and absolutely dominant. Finally, the Pauli blocking of the $\pi N$ decay reduces the $\Gamma_{\Delta}$ free width which now reads as

$$
\Gamma_{\Delta}^{\mathrm{Pauli}}=\Gamma_{\Delta} \frac{I_{1}+I_{2}}{2}
$$

The angular integrals $I_{1}$ and $I_{2}$ can be found in Appendix B of Ref. 51]. This selfenergy is taken into account by making the substitutions $M_{\Delta} \rightarrow M_{\Delta}+\operatorname{Re} \Sigma_{\Delta}$ and $\Gamma_{\Delta} / 2 \rightarrow \Gamma_{\Delta}^{\text {Pauli }} / 2-\operatorname{Im} \Sigma_{\Delta}$ in the propagator of Eq. (13).

\section{Cross section}

The hadronic current is further modified in the nucleus, where the nucleons are bound and thus have a momentum distribution. In the impulse approximation, and after summing over all nucleons, we can write it as

$$
J_{N \pi}^{\mu}=-\frac{i}{2} \int d^{3} r e^{i\left(\vec{q}-\vec{p}_{\pi}\right) \cdot \vec{r}}\left[\rho_{p}(r)+\frac{\rho_{n}(r)}{3}\right] \sqrt{3} \frac{f^{*}}{m_{\pi}} F\left(p^{\prime}\right) \tilde{D}\left(p^{\prime}\right) p_{\pi}^{\alpha} \operatorname{Tr}\left\{\bar{u}(0) \Lambda_{\alpha \beta} \mathcal{A}^{\beta \mu} u(0)\right\},
$$

where $\tilde{D}\left(p^{\prime}\right)$ is the in-medium $\Delta$ propagator and the trace corresponds to the sum over the nucleons' spin. In the evaluation of the trace we have taken an average nucleon momentum for the spinors neglecting corrections of order $(p / M)^{2}$. Given the large mass of the nucleus, the pion energy is taken to be equal to $q^{0}=E_{\nu}-E_{\mu}$ and this defines the modulus of the asymptotic pion momentum. On the other hand, a momentum of $\vec{q}-\vec{p}_{\pi}$ is transferred to the nucleus. In the evaluation of the amplitude we assume that this momentum is equally shared by the initial and the final nucleon, so that they have $\vec{p}_{i}=\left(\vec{p}_{\pi}-\vec{q}\right) / 2$ and $\vec{p}_{f}=\left(\vec{q}-\vec{p}_{\pi}\right) / 2$ respectively. This prescription has also been used in Refs. [57, 61] for coherent $\pi^{0}$ photo- and electroproduction. The approximation is based on the fact that, for Gaussian nuclear wave functions, it leads to an exact treatment of the terms linear in momentum of the elementary amplitude and allows for a consistent description of the pion-nucleon and pion-nucleus kinematics [61]. Pion distortion is taken into account by the replacements

$$
e^{-i \vec{p}_{\pi} \cdot \vec{r}} \rightarrow \phi_{\text {out }}^{*}\left(\vec{p}_{\pi}, \vec{r}\right)
$$

and

$$
\vec{p}_{\pi} e^{-i \vec{p}_{\pi} \cdot \vec{r}} \rightarrow i \vec{\nabla} \phi_{\text {out }}^{*}\left(\vec{p}_{\pi}, \vec{r}\right)
$$

where $\phi_{\text {out }}^{*}\left(\vec{p}_{\pi}, \vec{r}\right)$ is an outgoing solution of the KG equation for a pion of asymptotic momentum $\vec{p}_{\pi}$ calculated with the optical potential described below. To test the validity of the eikonal approximation we have also calculated the cross section with the pion wave function

$$
\phi_{\text {out }}^{*, e i k}\left(\vec{p}_{\pi}, \vec{r}\right)=e^{-i \vec{p}_{\pi} \cdot \vec{r}} e^{-i \int_{z}^{\infty} \frac{\Pi\left(\rho\left(\vec{b}, z^{\prime}\right)\right)}{2 p_{\pi}} d z^{\prime}},
$$


where $\vec{r}=(\vec{b}, z)$ and $\Pi$ is the pion selfenergy described in the next section.

The cross section for the coherent process $\nu+A \rightarrow A+\mu^{-}+\pi^{+}$is then given by

$$
\frac{d \sigma}{d \Omega_{\ell} d E_{\ell} d \Omega_{\pi}}=\frac{1}{8} \frac{\left|\vec{k}^{\prime}\right|\left|\vec{p}_{\pi}\right|}{|\vec{k}|} \frac{1}{(2 \pi)^{5}}|\mathcal{M}|^{2},
$$

with

$$
\mathcal{M}=\frac{G}{\sqrt{2}} \cos \theta_{c} l_{\alpha} J_{N \pi}^{\alpha}
$$

\section{Pion optical potential and distorted wave function}

The pion wave function is the solution of the KG equation with an optical potential. Since most of the produced pions lie in the energy region around the $\Delta$ excitation the $\Delta$-hole model can be used. In this model, which we take from Ref. [50], the optical potential is given by

$$
V_{o p t}=\frac{\Pi}{2 \omega},
$$

where $\omega$ is the pion energy in the laboratory system and the selfenergy $\Pi$ reads as

$$
\Pi=-4 \pi \frac{M^{2}}{s} \vec{q}^{2} \frac{\mathcal{P}}{1+4 \pi g^{\prime} \mathcal{P}} .
$$

Here, $s$ is the Mandelstam variable of the $\pi N$ system and $\vec{q}$ is the pion laboratory momentum. Finally, $\mathcal{P}$ is given by

$$
\mathcal{P}=-\frac{1}{6 \pi}\left(\frac{f^{*}}{m_{\pi}}\right)^{2}\left\{\frac{\rho_{p}+\rho_{n} / 3}{\sqrt{s}-M_{\Delta}-\operatorname{Re} \Sigma_{\Delta}^{0}+i \Gamma_{\Delta}^{\mathrm{Pauli}} / 2-i \operatorname{Im} \Sigma_{\Delta}}+\frac{\rho_{n}+\rho_{p} / 3}{-\sqrt{s}-M_{\Delta}+2 M-\operatorname{Re} \Sigma_{\Delta}^{0}}\right\},
$$

where the two terms correspond to direct and crossed $\Delta$-hole excitations. The neutron densities $\rho_{n}$ are taken from Ref. [62] and the proton densities $\rho_{p}$ are obtained from the parameterizations compiled in Ref. [63], both deconvoluted to take into account the finite size of the nucleons as done in [62]. The real and imaginary part of the $\Delta$ selfenergy $\Sigma_{\Delta}$ and the Pauli corrected width, $\Gamma_{\Delta}^{\text {Pauli }}$, have been described in section $\amalg$ B

In coordinate space and for finite nuclei, this p-wave potential can be cast as

$$
2 \omega V_{\text {opt }}(\vec{r})=4 \pi \frac{M^{2}}{s}\left[\vec{\nabla} \cdot \frac{\mathcal{P}(r)}{1+4 \pi g^{\prime} \mathcal{P}(r)} \vec{\nabla}-\frac{1}{2} \frac{\omega}{M} \Delta \frac{\mathcal{P}(r)}{1+4 \pi g^{\prime} \mathcal{P}(r)}\right],
$$

where the first term has the standard Kisslinger form and the second one accounts for the angular transformation from center-of-mass to laboratory variables. The $r$ dependence in $\mathcal{P}$ appears via the local density approximation $\rho \rightarrow \rho(r)$. With this potential we solve the KG equation and obtain the pion scattering wave function. The procedure is described in detail in Refs. [51, 64]. To asses the quality of the potential one can compare its results for differential cross sections with pion nucleus elastic scattering data. We obtain an overall good agreement from light to heavy nuclei at the energies relevant for this work. We can also refer the reader to the article by Garcia Recio et al. [65], where a very similar potential was considered, using as here the local density approximation and the same values for the $\Delta$ selfenergy. The main differences with that work are their inclusion of a phenomenological s-wave selfenergy, and our inclusion of the $\Delta$ crossed term. We have checked that both pieces produce only minor effects in the $\Delta$ resonance region.

\section{RESULTS}

We show in Fig. 1 the differential cross section $d \sigma / d p_{\pi}$ for $\mathrm{CC}$ coherent pion production in ${ }^{12} \mathrm{C}$ at a neutrino energy $E_{\nu}=1 \mathrm{GeV}$. This plot shows the effect of the different ingredients of the calculation. The modification of the elementary reaction mechanism, through the inclusion of the $\Delta$ selfenergy in the propagator, already produces a strong reduction of the cross section. This reduction of around 35\% agrees with the results of Ref. [19]. The pion distortion further decreases the cross section and moves the peak to lower energies. This reflects the presence of a strongly absorptive part in the optical potential around the $\Delta$ resonance peak. The final result shows that the pion 


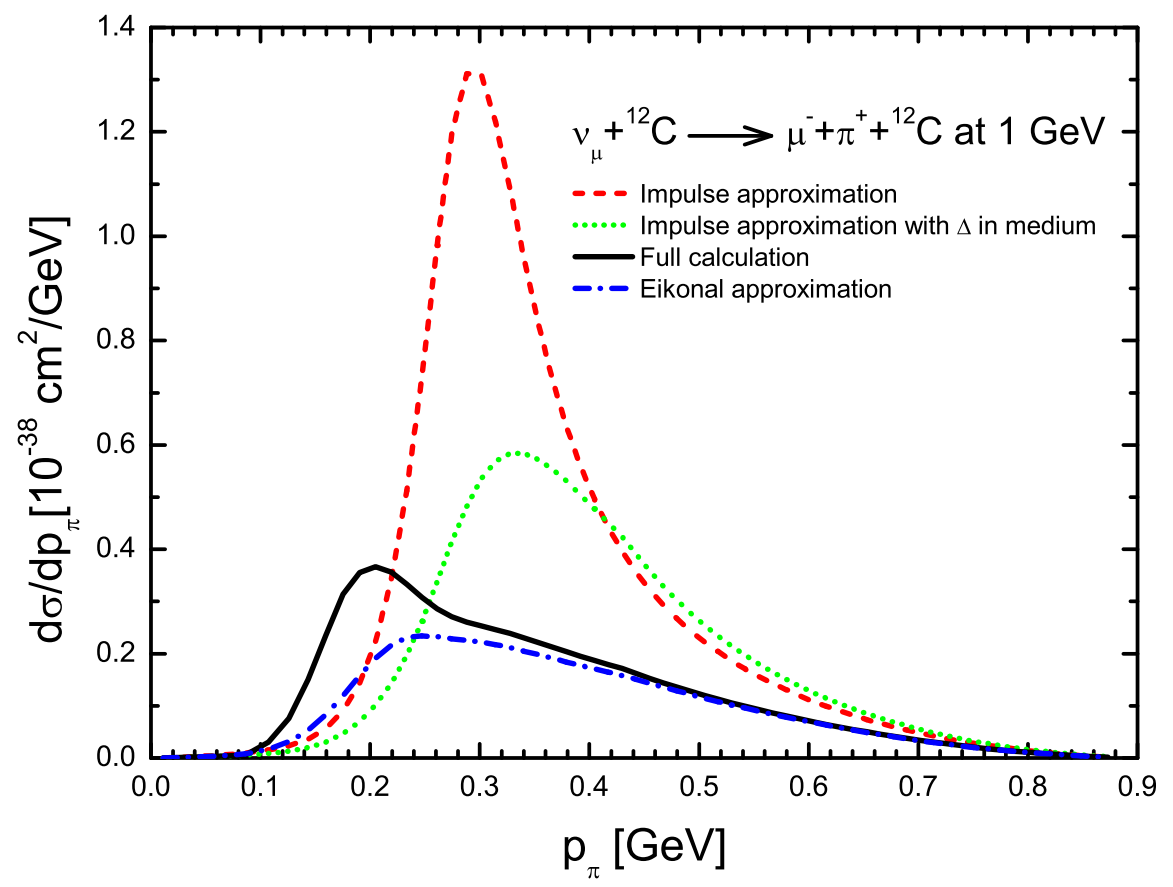

FIG. 1: (Color online) Momentum distribution of the coherent pions.

spectrum is peaked at much lower energies or, equivalently, that the muon energy distribution is peaked at higher energies than for the impulse approximation. As expected, the eikonal approximation fails for low and intermediate energies, where a better treatment of the pion wave function is clearly required. Our eikonal result differs from the one obtained in Ref. [19] because of their use of the asymptotic momentum in the amplitude, whereas we take the gradient of the distorted pion wave function, and our more complete treatment of the optical potential (see Eq. (26) vs. Eq. (6) of Ref. [19]).

In Fig. 2] we present the same observable averaged over the K2K [66] and the MiniBooNE [67] spectra compared with the results for a fixed neutrino energy. The consideration of high energy neutrinos widens the pion momentum distribution, due to phase space. Even when both spectra are quite different and K2K has a larger average neutrino energy $(1.3 \mathrm{GeV})$ than MiniBooNE $(0.75 \mathrm{GeV})$, the peak position stays at the same pion momentum, below the $\Delta$ resonance. Furthermore, most of the pions have relatively low energies such that the use of the $\Delta$-hole model is appropriate.

Although the total cross section and the energy distribution are strongly modified by the nuclear medium, the angular distribution of the muons remains relatively unaffected, as can be seen on the left panel of Fig. 3 where we compare the result of our model with the impulse approximation, rescaled to match the full calculation at zero degrees. On the right panel, the muon angular distributions averaged over the K2K and the MiniBooNE neutrino spectra are shown together with the one obtained for $1 \mathrm{GeV}$ neutrinos. In both cases, the consideration of higher energy neutrinos leads to a narrower angular distribution. As a consequence, the MiniBooNE angular distribution is appreciably more forward peaked than the one at $1 \mathrm{GeV}$ even when its average energy is lower. We have also checked that the lower bound on the muon energy used by K2K in Ref. 2] $\left(p_{\mu}>450 \mathrm{MeV} / \mathrm{c}\right)$ does not modify appreciably this observable.

We show the total cross section as a function of the neutrino energy and for several nuclei in Fig. 4, with the caveat that the pion production model is less satisfactory at high energies. One reason is that mechanisms, other than the excitation of the $\Delta$ resonance, become relevant. Also the pion distortion, based on the $\Delta$-hole model, is not appropriate for the high energy pions that can be produced by neutrinos with $E_{\nu}>2 \mathrm{GeV}$. Nonetheless, the $\pi N$ interaction is much weaker at high energies than at the $\Delta$ peak and thus distortion effects should be smaller there.

In Fig. 5. we present the dependence of the total cross section on the atomic number. In this process, the amplitude is the coherent sum of the contributions of all participant nucleons. Taking into account the isospin factors, this implies that the amplitude is proportional to an effective number of participants defined as $\mathrm{P}=\mathrm{Z}+\mathrm{N} / 3$; here $\mathrm{Z}$ and $\mathrm{N}$ are the 


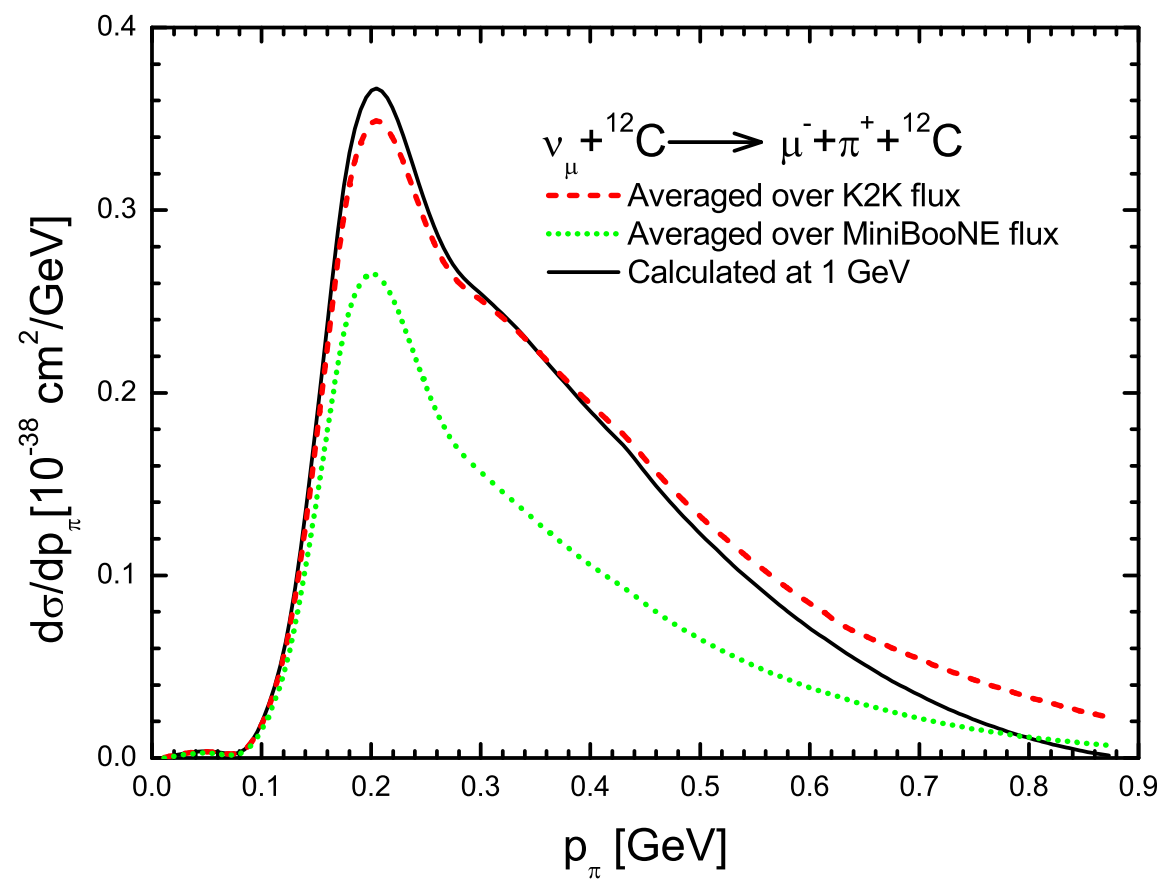

FIG. 2: (Color online) Momentum distribution of the coherent pions averaged over the K2K and MiniBooNE fluxes; the same distribution at $E_{\nu}=1 \mathrm{GeV}$ is also plotted for reference.

number of protons and neutrons respectively. This could suggest a quadratic dependence of the cross section on $\mathrm{P}$, so that for heavier nuclei, the process could be comparatively larger with respect to incoherent $\pi$ production or other processes. However, there are several reasons that quench the $\mathrm{P}$ dependence. First, pion absorption is quite strong and forces the reaction to be peripheral. Notice that the cross sections for the inclusive processes are not affected by this. Second, the nuclear form factor is narrower for heavy nuclei, and reduces more the contribution from high momentum transfers.

Our result for the integrated cross section for coherent pion production on ${ }^{12} \mathrm{C}$ averaged over the $\mathrm{K} 2 \mathrm{~K}$ flux is $\sigma_{\text {coh }}^{C C}=10 . \times 10^{-40} \mathrm{~cm}^{2}$. This value is above the upper limit of $7.7 \times 10^{-40} \mathrm{~cm}^{2}$ obtained using the ratio between coherent and $\sigma^{C C}$, the total CC cross section, from the K2K collaboration [2] and the value for $\sigma^{C C}$ of their MC calculation. Without the experimental threshold for the muon momentum, $p_{\mu}>450 \mathrm{MeV} / c$, we obtain $\sigma_{c o h}^{C C}=12 . \times 10^{-40} \mathrm{~cm}^{2}$.

There are several factors that might conspire to produce the disagreement between our calculation and the experimental upper bound. First of all, due to the considerable uncertainty in the experimental data for pion production cross sections on the nucleon, the axial N- $\Delta$ form factors are not sufficiently constrained. A more complete theoretical description of the elementary amplitude with the inclusion of background terms [23, 40] and heavier resonances [23, 39] could help, but in order to put such a model on a firm ground, more precise data are required. The optical potential employed in our calculation is realistic around the $\Delta$ peak (i.e. for pions with $150-450 \mathrm{MeV} / \mathrm{c}$ momenta), where most of the strength of the reaction actually concentrates, but has room for improvement both at lower and higher energies.

It is also important to recall that at forward angles, where the coherent process is sizable, nuclear effects play an important role and this may affect the experimental separation of the coherent events from the incoherent ones which, to a large extent, relies on the theoretical models built in the MC simulations. The situation is illustrated in Fig. 6] where we plot the muon angular distributions averaged over the $\mathrm{K} 2 \mathrm{~K}$ flux (and with $p_{\mu}>450 \mathrm{MeV} / \mathrm{c}$ ) for coherent $\pi^{+}$production, together with the main contributions to the total inclusive CC cross section: quasielastic scattering $(\mathrm{QE})$ and incoherent $\Delta$ excitation. The calculations of the $\Delta$ part is performed with the same elementary amplitudes and in-medium effects as given above for the coherent reaction. For the quasielastic process, we have adopted the model of Ref. [68] but updating the nucleon form factors according to [69]. Nuclear effects include Fermi motion, Pauli blocking with a local Fermi gas and the renormalization of the weak transition, which is treated as an RPA resummation of particle-hole and $\Delta$-hole states. These nuclear correlations cause a considerable reduction of strength at low $q^{2}$ (forward angles), as can be seen in Fig. 6. while they are negligible for $\cos \theta_{\mu}<0.8$. Therefore, if a 

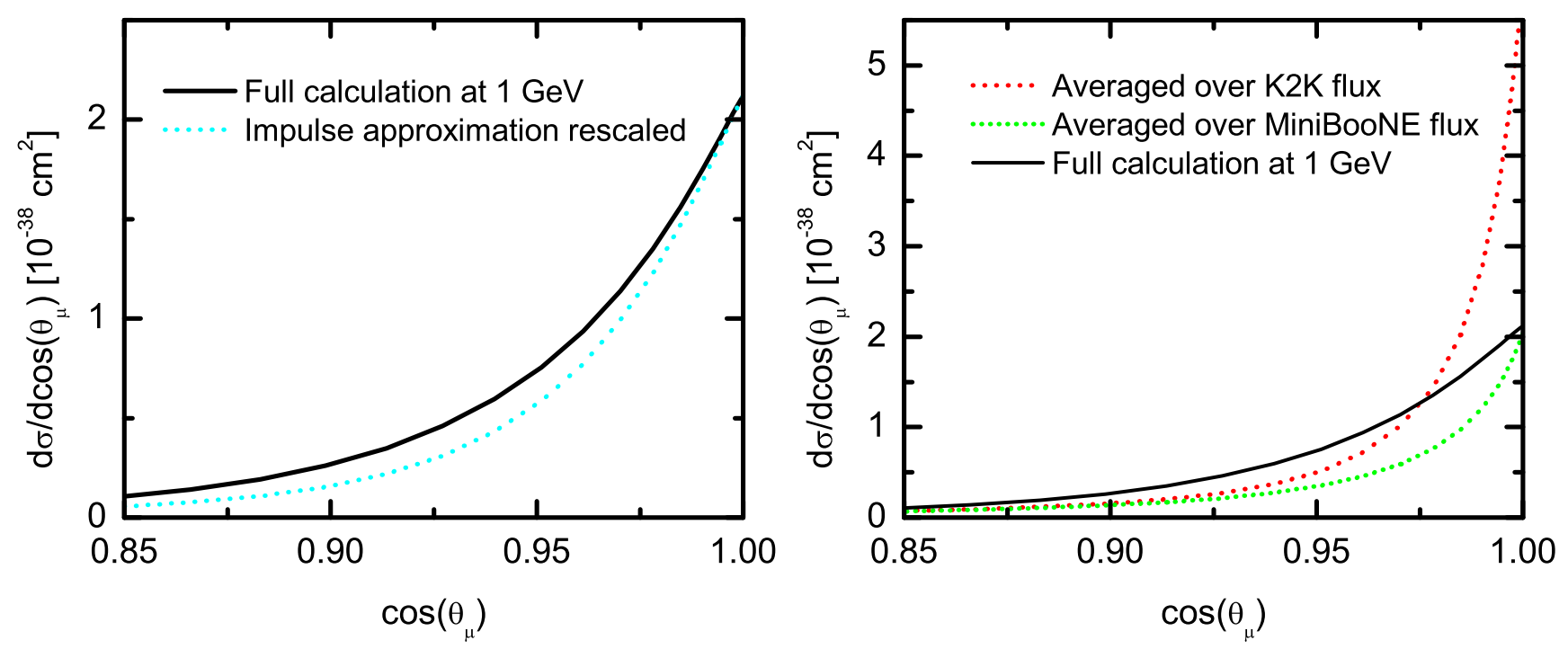

FIG. 3: (Color online) Muon angular distribution in CC coherent pion production. Left panel: full model vs. impulse approximation. The latter has been rescaled to match the full calculation at zero degrees. Right panel: full model at $E_{\nu}=1$ $\mathrm{GeV}$ and averages over $\mathrm{K} 2 \mathrm{~K}$ and MiniBooNE fluxes.

model that lacks these correlations is used to extrapolate the data from the region of $\cos \theta_{\mu} \lesssim 0.8$ to forward angles, one might overestimate the QE part, causing an underestimation of the contribution of other mechanisms, like the coherent pion production, to the cross section.

\section{SUMMARY AND CONCLUSIONS}

We have studied CC coherent pion production induced by muon neutrinos $\nu_{\mu}+A \rightarrow \mu^{-}+\pi^{+}+A$. Our model takes into account the modification of the production mechanism due to the renormalization of the $\Delta$ properties in the nuclear medium and the distortion of the final pion. The distorted pion wave function is obtained by solving the Klein Gordon equation with an optical potential based on the $\Delta$-hole model. Both effects produce a large reduction of the cross section with respect to the impulse approximation. The distortion of the pion shifts the peak of the pion energy distributions towards low energies. The angular distributions are slightly widened by the nuclear medium effects.

The A dependence of the coherent process has been investigated. We have found that the integrated cross section grows more slowly than one would naively expect due to the strong pion absorption and the effect of nuclear form factors. Therefore, we do not expect that coherent pion production becomes more relevant with respect to the incoherent processes for heavier nuclei.

We have also studied the cross sections averaged over the K2K and MiniBooNE spectra. In the case of K2K, we find a cross section $30 \%$ larger than the upper limit estimated in the experiment. One should however remember that such upper limit is based on values for the $\mathrm{CC}$ cross sections on nuclei (quasielastic, pion production, etc.) which are not well known at the rather low energies discussed here. Further improvements in the theoretical description of this reaction will require more precise data for the neutrino nucleon cross sections at low energies that could constrain the axial N- $\Delta$ form factors. As for MiniBooNE, we give predictions for energy and angular distributions which can be useful to compare with and analyze their data.

\section{Acknowledgments}

We thank P. Novella and J. J. Gomez-Cadenas for useful discussions. This work was partially supported by DGI and FEDER funds, contract BFM2003-00856, by the EU Integrated Infrastructure Initiative Hadron Physics Project contract RII3-CT-2004-506078 and by the Research Cooperation Program of Japan Society for the Promotion of 


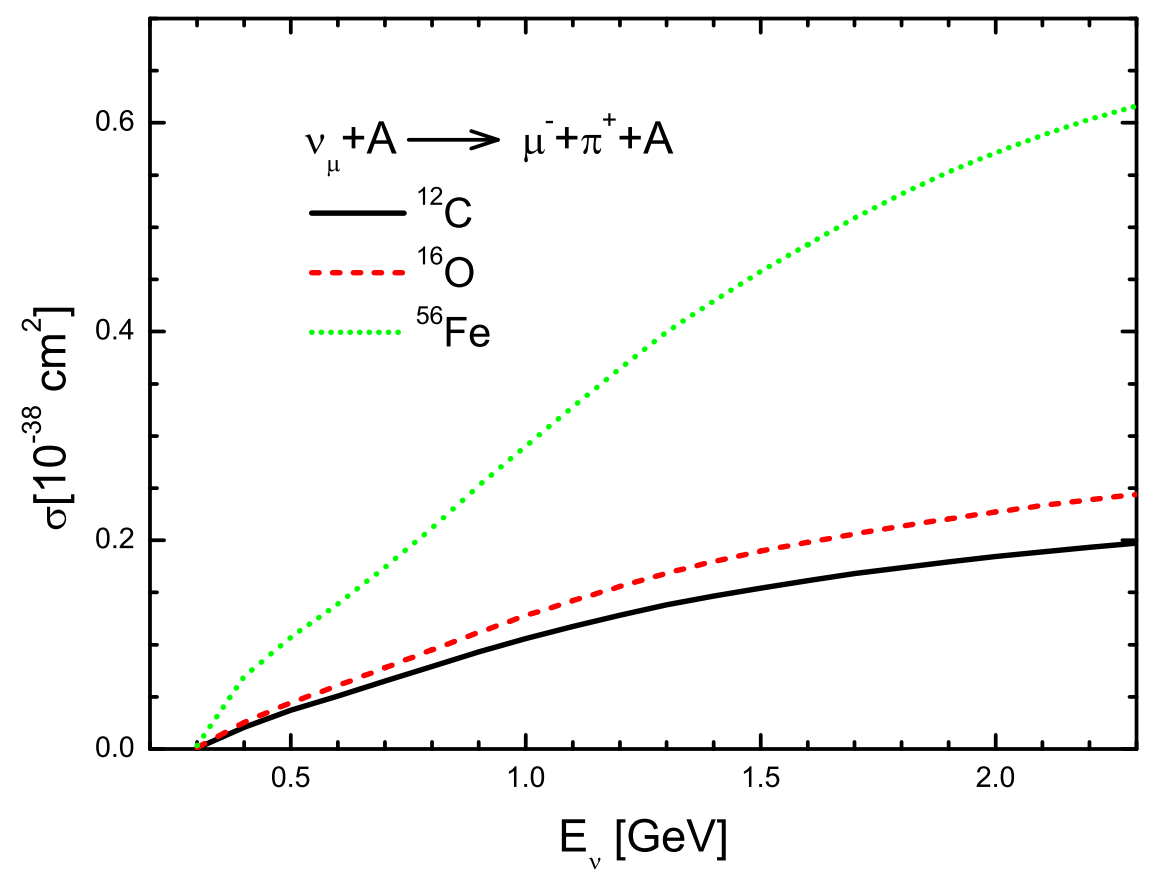

FIG. 4: (Color online) Total cross section for CC coherent pion production as a function of the neutrino energy for several nuclei.

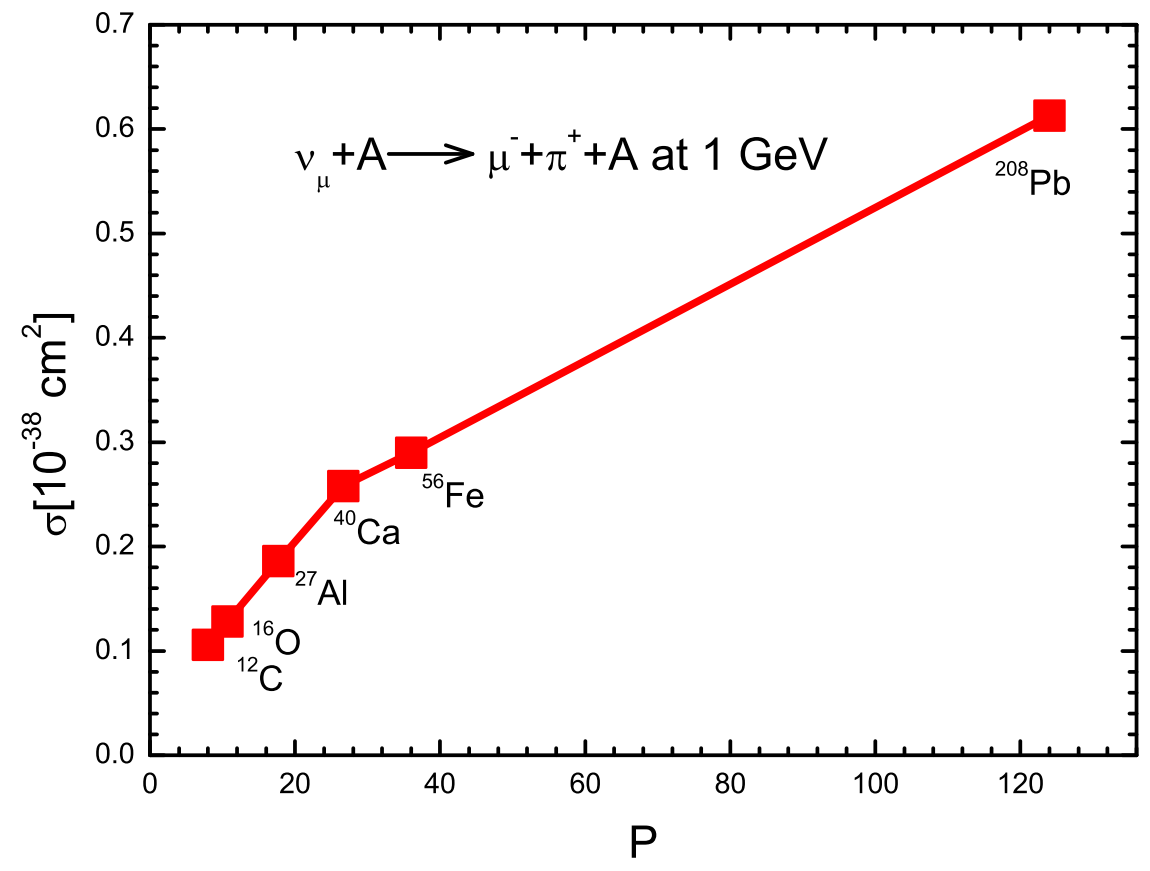

FIG. 5: (Color online) Total cross section for CC coherent pion production at $E_{\nu}=1 \mathrm{GeV}$ as a function of the effective number of participants $\mathrm{P}=\mathrm{Z}+\mathrm{N} / 3$, as explained in the text. 


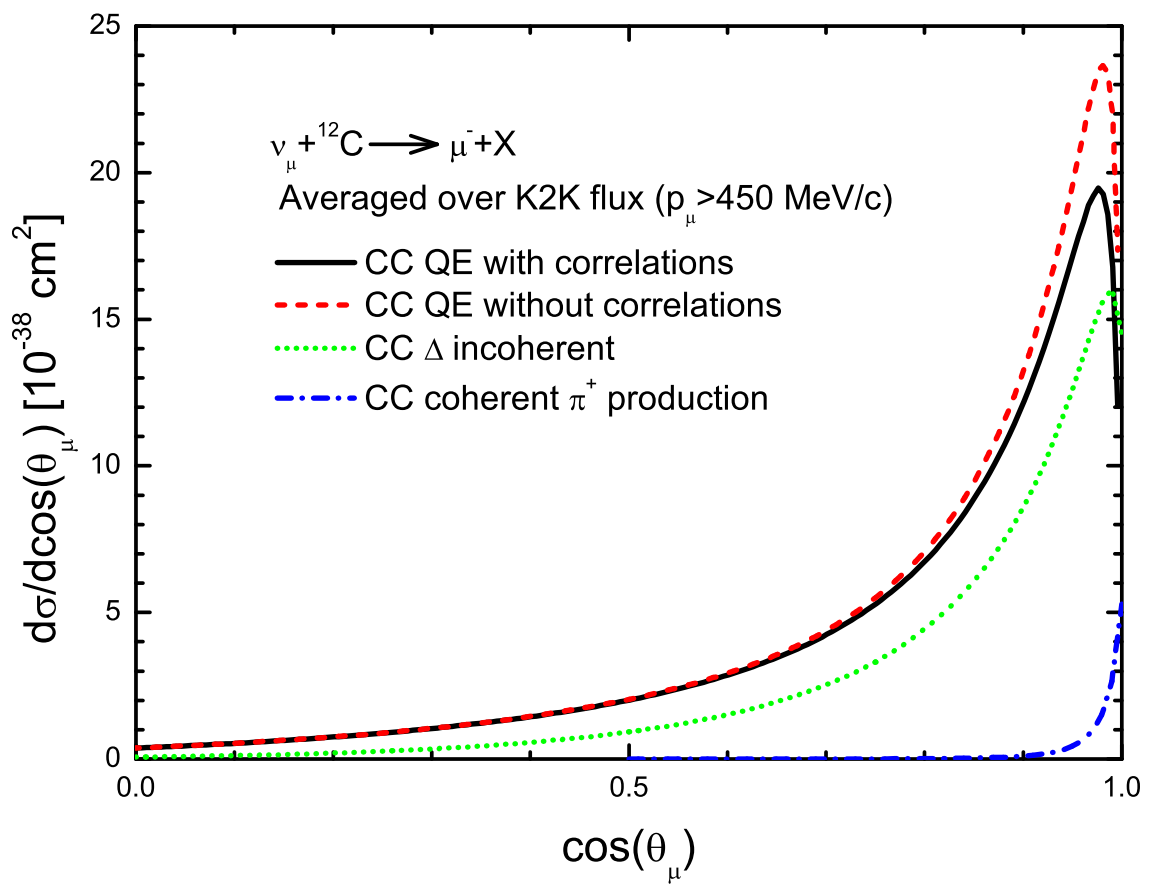

FIG. 6: (Color online) Muon angular distribution averaged over the K2K flux for incoherent CC quasielastic (QE) scattering, $\Delta$ excitation and coherent $\pi^{+}$production.

Science (JSPS) and Spanish CSIC.

[1] M. H. Ahn et al. [K2K Collaboration], Phys. Rev. Lett. 90 (2003) 041801.

[2] M. Hasegawa et al. [K2K Collaboration], Phys. Rev. Lett. 95 (2005) 252301.

[3] D. Rein and L. M. Sehgal, Nucl. Phys. B 223, 29 (1983).

[4] J. L. Raaf [BooNE Collaboration], Nucl. Phys. Proc. Suppl. 139 (2005) 47.

[5] D. Casper, Nucl. Phys. Proc. Suppl. 112 (2002) 161.

[6] H. Gallagher, Nucl. Phys. Proc. Suppl. 112 (2002) 188.

[7] Y. Hayato, Nucl. Phys. Proc. Suppl. 112 (2002) 171.

[8] M. O. Wascko [MiniBooNE Collaboration], Nucl. Phys. Proc. Suppl. 159, 50 (2006).

[9] H. J. Grabosch et al. [SKAT Collaboration], Z. Phys. C 31 (1986) 203.

[10] P. Marage et al. [BEBC WA59 COLLABORATION Collaboration], Z. Phys. C 31 (1986) 191.

[11] P. P. Allport et al. [BEBC WA59 Collaboration], Z. Phys. C 43 (1989) 523.

[12] M. Aderholz et al. [E632 Collaboration], Phys. Rev. Lett. 63 (1989) 2349.

[13] S. Willocq et al. [E632 Collaboration], Phys. Rev. D 47 (1993) 2661.

[14] P. Vilain et al. [CHARM-II Collaboration], Phys. Lett. B 313 (1993) 267.

[15] A. A. Belkov and B. Z. Kopeliovich, Sov. J. Nucl. Phys. 46 (1987) 499 [Yad. Fiz. 46 (1987) 874].

[16] E. A. Paschos, A. Kartavtsev and G. J. Gounaris, Phys. Rev. D 74 (2006) 054007.

[17] H. C. Kim, S. Schramm and C. J. Horowitz, Phys. Rev. C 53 (1996) 3131.

[18] N. G. Kelkar, E. Oset and P. Fernandez de Cordoba, Phys. Rev. C 55 (1997) 1964.

[19] S. K. Singh, M. Sajjad Athar and S. Ahmad, Phys. Rev. Lett. 96 (2006) 241801.

[20] S. Ahmad, M. Sajjad Athar and S. K. Singh, Phys. Rev. D 74 (2006) 073008.

[21] G. M. Radecky et al., Phys. Rev. D 25 (1982) 1161 [Erratum-ibid. D 26 (1982) 3297].

[22] T. Kitagaki et al., Phys. Rev. D 34 (1986) 2554.

[23] G. L. Fogli and G. Nardulli, Nucl. Phys. B 160 (1979) 116.

[24] H. C. Kim, S. Schramm and C. J. Horowitz, Phys. Rev. C 53 (1996) 2468.

[25] S. K. Singh, M. J. Vicente Vacas and E. Oset, Phys. Lett. B 416 (1998) 23 [Erratum-ibid. B 423 (1998) 428]. 
[26] L. Alvarez-Ruso, S. K. Singh and M. J. Vicente Vacas, Phys. Rev. C 59 (1999) 3386.

[27] T. Sato, D. Uno and T. S. H. Lee, Phys. Rev. C 67 (2003) 065201.

[28] O. Lalakulich and E. A. Paschos, Phys. Rev. D 71 (2005) 074003.

[29] T. Leitner, L. Alvarez-Ruso and U. Mosel, Phys. Rev. C 73 (2006) 065502.

[30] P. A. Schreiner and F. Von Hippel, Nucl. Phys. B 58 (1973) 333.

[31] L. Alvarez-Ruso, S. K. Singh and M. J. Vicente Vacas, Phys. Rev. C 57 (1998) 2693.

[32] T. Kitagaki et al., Phys. Rev. D 42 (1990) 1331.

[33] S. J. Barish et al., Phys. Rev. D 19 (1979) 2521.

[34] P. Allen et al. [Aachen-Bonn-CERN-Munich-Oxford Collaboration], Nucl. Phys. B 176 (1980) 269.

[35] J. Bell et al., Phys. Rev. Lett. 41 (1978) 1012.

[36] P. A. Zucker, Phys. Rev. D 4 (1971) 3350.

[37] J. Bijtebier, Nucl. Phys. B 21 (1970) 158.

[38] L. Tiator, D. Drechsel, S. Kamalov, M. M. Giannini, E. Santopinto and A. Vassallo, Eur. Phys. J. A 19 (2004) 55.

[39] O. Lalakulich, E. A. Paschos and G. Piranishvili, Phys. Rev. D 74 (2006) 014009.

[40] E. Hernandez, J. Nieves and M. Valverde, arXiv:hep-ph/0701149.

[41] M. Benmerrouche, R. M. Davidson and N. C. Mukhopadhyay, Phys. Rev. C 39 (1989) 2339.

[42] G. Penner and U. Mosel, Phys. Rev. C 66 (2002) 055211.

[43] L. S. Kisslinger and W. L. Wang, Phys. Rev. Lett. 30 (1973) 1071.

[44] M. Hirata, J. H. Koch, E. J. Moniz and F. Lenz, Annals Phys. 120 (1979) 205.

[45] E. Oset and W. Weise, Nucl. Phys. A 329 (1979) 365.

[46] Y. Horikawa, M. Thies and F. Lenz, Nucl. Phys. A 345 (1980) 386.

[47] E. Oset, H. Toki and W. Weise, Phys. Rept. 83, 281 (1982).

[48] R. A. Freedman, G. A. Miller and E. M. Henley, Nucl. Phys. A 389 (1982) 457.

[49] M. Hirata, F. Lenz and M. Thies, Phys. Rev. C 28 (1983) 785.

[50] E. Oset and L. L. Salcedo, Nucl. Phys. A 468 (1987) 631.

[51] J. Nieves, E. Oset and C. Garcia-Recio, Nucl. Phys. A 554 (1993) 554.

[52] L. L. Salcedo, E. Oset, M. J. Vicente-Vacas and C. Garcia-Recio, Nucl. Phys. A 484 (1988) 557.

[53] M. J. Vicente Vacas and E. Oset, Nucl. Phys. A 568 (1994) 855.

[54] R. C. Carrasco and E. Oset, Nucl. Phys. A 536 (1992) 445.

[55] R. C. Carrasco, E. Oset and L. L. Salcedo, Nucl. Phys. A 541 (1992) 585.

[56] A. Gil, J. Nieves and E. Oset, Nucl. Phys. A 627 (1997) 543.

[57] R. C. Carrasco, J. Nieves and E. Oset, Nucl. Phys. A 565 (1993) 797.

[58] S. Hirenzaki, J. Nieves, E. Oset and M. J. Vicente-Vacas, Phys. Lett. B 304, 198 (1993).

[59] P. Fernandez de Cordoba, J. Nieves, E. Oset and M. J. Vicente-Vacas, Phys. Lett. B 319 (1993) 416.

[60] P. Fernandez de Cordoba, E. Oset and M. J. Vicente-Vacas, Nucl. Phys. A 592 (1995) 472.

[61] D. Drechsel, L. Tiator, S. S. Kamalov and S. N. Yang, Nucl. Phys. A 660, 423 (1999).

[62] C. Garcia-Recio, J. Nieves and E. Oset, Nucl. Phys. A 547 (1992) 473.

[63] C. W. De Jager, H. De Vries and C. De Vries, Atom. Data Nucl. Data Tabl. 36 (1987) 495.

[64] J. Nieves, E. Oset and C. Garcia-Recio, Nucl. Phys. A 554 (1993) 509.

[65] C. Garcia-Recio, E. Oset, L. L. Salcedo, D. Strottman and M. J. Lopez, Nucl. Phys. A 526, 685 (1991).

[66] M. H. Ahn et al. [K2K Collaboration], Phys. Rev. D 74 (2006) 072003.

[67] J. Monroe [MiniBooNE Collaboration], Nucl. Phys. Proc. Suppl. 139 (2005) 59.

[68] S. K. Singh and E. Oset, Nucl. Phys. A 542 (1992) 587.

[69] H. Budd, A. Bodek and J. Arrington, arXiv:hep-ex/0308005 